\title{
Intervención de órganos del Estado en resolución de conflictos de usuarios de Agua Virú, 2021
}

Intervention of State Organs in Conflict Resolution of Users of Agua Virú, 2021

\author{
Zurit Fiorela Yakuden Polonio, ${ }^{a}$ fiorelayakudenpolonio@gmail.com \\ ORCID: https://orcid.org/0000-0002-7642-311X \\ Universidad César Vallejo \\ Carlos Marvin Machado Peralta, ${ }^{\mathrm{b}}$ carlosmachadoperalta@gmail.com \\ ORCID: https://orcid.org/0000-0001-5244-0145 \\ Universidad César Vallejo
}

Recibido: Octubre / 05 /2021 - Revisado: Noviembre /25 /2021 - Publicado: Diciembre /29 / 2021

\section{RESUMEN}

La presente investigación manifiesta la forma que interviene la Autoridad Nacional del Agua en la resolución de conflictos por el recurso hídrico que presentaron los usuarios agrícolas de Virú, pues como se tiene de conocimiento la cuenca del Santa y Virú es la que provee de agua a la ciudad de Virú para atender los servicios básicos como uso poblacional, industrial y agrícola. Este estudio tuvo como objetivo analizar la intervención de órganos supervisores del Estado en la resolución de conflictos de los usuarios agrícolas de Agua Virú - 2021, y que estrategias usan para llegar a acuerdos; incluyendo la Ley de Recursos Hídricos y la Ley de Organizaciones de Usuarios. Para llevar a cabo esta investigación, se realizaron entrevistas semiestructuradas a los participantes que accedieron, se revisó información de otros trabajos y se entregaron cuestionarios a los usuarios para que puedan manifestar su punto de vista. La conclusión de este estudio es que la intervención de ANA en la resolución de conflictos es efectiva y viene aplicando correctamente la Ley de Recursos Hídricos y la Ley de Organización de Usuarios en el marco de la legitimidad y transparencia.

Palabras claves: Intervención de órganos del estado, resolución de conflictos.

\begin{abstract}
In the present investigation, it shows the way in which the National Water Authority intervenes in the resolution of conflicts over the water resource presented by the agricultural users of Virú, since, as is known, the Santa y Virú basin is the one that provides water to the city of Virú to serve basic services such as population, industrial and agricultural use. The objective of this study was to analyze the intervention of State supervisory bodies in the resolution of conflicts of agricultural users of Agua Virú - 2021, and what strategies they use to reach agreements; including the Law on Water Resources and the Law on User Organizations. To carry out this research, semi-structured interviews were conducted with the participants who agreed, information from other works was reviewed and questionnaires were given to users so that they can express their point of view. The conclusion of this study is that ANA's intervention in conflict resolution is effective and has been correctly applying the Water Resources Law and the User Organization Law within the framework of legitimacy and transparency.
\end{abstract}

Keywords: Intervention of state bodies, conflict resolution. 


\section{$\underline{\text { Introducción }}$}

En la gestión ambiental y territorial que implica la resolución de controversias, los elementos de $\mathrm{H} 2 \mathrm{O}$ en continentes como África son escasos e insuficientes para abastecer a toda la población de la región. Por eso intervienen los organismos reguladores y exponen a los usuarios en asuntos que deben ser decididos. El espacio de gestión que brinda la Gestión Integrada de los Recursos Hídricos (GIRH) es cuenca, más aún, es el modelo de gestión del agua que ha adoptado nuestro país.

En Perú, la agricultura de regadío crea el valor económico de la producción agrícola, que es de gran importancia para satisfacer indispensables necesidades humanas y promover el avance económico de la mayoría de los países en progreso; la superficie agrícola es de 6.480 millones de hectáreas (39\% del área agrícola total), La región Libertad tiene 433.000 hectáreas (que representan el 6,69\% de la superficie agrícola nacional). (Anuario de Estadísticas Ambientales 2017). Esta es la actividad más económica para los trabajadores peruanos; entre ellos, al 30 de abril de 2017, la población económicamente activa (PEA) total era de 17,5 millones, de los cuales los hombres representaban el 56,2\% y las mujeres el 43,8\%. (Instituto Nacional de Estadística e Informática-INEI).

Este tema es singularmente significativo en la cuenca del rio Santa, ubicado entre los departamentos de La Libertad y Ancash, a los que se encarga de proveer agua, en dichas regiones habitan aproximadamente 2.9 millones de personas (INEI,2017), un número considerable del país.

Los habitantes del distrito de Virú deben proporcionar diversos bienes y servicios (alimentación, educación, saneamiento, agua y saneamiento, fuentes de empleo y otros servicios básicos). Sin embargo, uno de los problemas para atender estas necesidades es que la mayor parte del territorio se ubica en zonas áridas, por lo que, como zona agrícola, se debe realizar un manejo estricto para satisfacer sus necesidades (costo, cobertura, planificación, previsión). Si bien Virú y Santa son las dos cuencas que abastecen de agua a la zona de Virú, la cuenca del Río Santa es un lugar de reunión para un gran número de los llamados usuarios agrícolas y no agrícolas (según la clasificación normativa), y también es el lugar donde se 
utiliza la cuenca del Virú para riego no regulado. Es por ello que la Junta de Usuarios del Sector Hidráulico Menor Virú, fue seleccionado para comprender y analizar cómo los usuarios del agua interactúan para cumplir con los diferentes requisitos para la provisión de recursos hídricos dentro del territorio de Virú, independientemente de si el país puede haberles asignado una cierta cantidad de derecho al agua. Por lo tanto, la investigación propuesta ayudará a comprender las herramientas implementadas por los entes del estado, la cual facilita la resolución de conflictos entre usuarios agrícolas por el recurso hídrico, da a conocer si han llegado a un acuerdo continuo en el tiempo; y analizar el rol que estos organismos supervisores del estado desempeñan a través de la normativa vigente representada por la Ley de Recursos Hídricos (2009), que formuló el modelo de Gestión Integrada de Recursos Hídricos (GIRH).

Esta problemática no lleva preguntar ¿de qué manera intervienen los órganos del Estado en resolución de conflictos de usuarios de Agua Virú? Entonces, el objetivo general fue analizar la intervención de órganos del Estado en resolución de conflictos. Desde el surgimiento de la humanidad, el agua se ha convertido en un recurso indispensable para la supervivencia y el desarrollo económico. A lo largo de los años, la población mundial y el desarrollo industrial han aumentado continuamente la demanda de agua, por lo que hoy la gente puede saber con certeza que el agua se está agotando. Altamirano (2018)

En este sentido, el agua es básica, no solo porque el cuerpo necesita agua para sobrevivir; sino a la vez, desarrollar algunas actividades que implican alguna lógica productiva y económica; o, según Bonilla (2020), como una especie de Esencial Recursos indispensables, sin él es imposible alcanzar estándares de desarrollo humano en la sociedad contemporánea.

Algunos estudios encontrados fueron los de Barrientos (2011) investigó sobre; Modelo de gestión integrada de recursos hídricos de las cuencas de los ríos Moquegua y Tambo, donde concluyó que el recurso hídrico es ineficiente en la zona, además indica que los volúmenes de agua otorgados en las licencias de agua son elevadas, los usuarios presentan alta morosidad en sus pagos, e indican poca o casi nula presencia de la Administración Local 
del Agua dentro de su jurisdicción por lo que recomiendan la implementación de las cuencas Moquegua y Tambo.

Jara (2019) investigó sobre Evaluación de la rentabilidad de los ingresos por uso con fines agrarios en la Autoridad Local del Agua Sicuani Periodo 2018. Se concluye que la Administración Local del Agua ha tomado malas decisiones al fiscalizar el cobro de la retribución económica que los usuarios deben cancelar al estado como derecho por el uso del agua, además que las aportaciones presentaron una tendencia desfavorable generando pérdidas considerables al estado, lo que indica que la entidad no ha tenido una buena política de gestión en esa zona.

Huaricallo (2014) investigó sobre Análisis de la gestión del recurso hídrico para consumo humano en la microcuenca Huancho, Huancane, Puno, Se concluyó que la operatividad de la Administración Local del Agua es limitada, la ley de recursos hídricos solo se aplica en un 40\%, el ALA tiene poca capacidad de gestión y presencia dentro de este sector al que el autor domina como actor marginado, además que se le recomienda contribuir con herramientas que faciliten obtener los derechos de uso de agua según su uso para las comunidades campesinas, de acuerdo a lo que establece el reglamento.

López (2018) investigó sobre Análisis de la intervención social y la calidad del recurso hídrico en el proyecto "Ampliación de los sistemas de agua potable y alcantarillado", Lurigancho, 2018, donde se concluyó que la intervención social era suficiente y la calidad del recurso era eficiente con un resultado correlacional según Rho de Spearman de 0.851, por lo que este autor revela que los usuarios estaban conformes con la gestión del recurso hídrico y la labor que realiza el ALA dentro de este territorio.

\section{Resolución de conflictos por el agua}

La intervención en la resolución de conflictos, y dos categorías las cuales son intervención de los órganos supervisores del estado con los usuarios agrícolas de Agua Virú2021. Oré y Rap (2009) en su trabajo de investigación Políticas neoliberales de agua en el Perú. Antecedentes y entretelones de la ley de recursos hídricos; La situación actual, desde 
el exterior, parece ser un incidente aislado, pero en conjunto conducen a la determinación de la coincidencia de las políticas de gestión de recursos hídricos espaciales públicos y privados impulsadas en el país, y la posterior adopción de la gestión integral de recursos hídricos en la Ley de Recursos Hídricos, desde Efectivo en 2009. Afirma que ANA es la agencia reguladora de más alta tecnología. El modelo prevé la creación de organizaciones integradas por países, usuarios y personas interesadas en la gestión, como comités de cuencas hidrográficas y organizaciones de usuarios, dejando atrás las organizaciones de riego y reguladas por la derogada Ley General de Aguas (1969).

Del Castillo (2009), en su investigación titulada; El régimen legal del agua, proporciona otra perspectiva sobre las percepciones cambiantes del agua a nivel mundial, desde verla como un recurso inagotable hasta verla como finita. Respecto a la región de los Andes, lo que se destaca es que la gestión es departamental, y está evolucionando hacia una gestión que reconoce que los recursos están integrados, por lo que los usuarios deben actuar en conjunto, lo que también destaca el cambio de espacio de gestión a cuenca en lugar de restricciones políticas. , muestra La evolución normativa desde la época del gobernador hasta la Ley del Agua (1969) incluye cambios constitucionales que cambiaron la visión de la gestión de recursos. También detalla la brecha entre la realidad y la Ley de Recursos Hídricos (LRH) a la fecha de publicación (2009), así como los cambios normativos que se han producido en la organización usuaria correspondiente (antes Comité de Usuarios de Riego)., incluido el registro de riego en el libro. Cambios estrechamente relacionados con el rol que juega la organización usuaria (antes organización de riego) en la práctica, como ayudar a resolver conflictos en los recursos nacionales, convirtiéndose en una organización privada, como se ve en este documento.

\section{Los recursos hídricos}

Sepulveda et al. (2020) El conflicto de los recursos hídricos debido a la mala calidad y disponibilidad de los recursos ha llevado a un acceso limitado y escaso. Esta situación limita el desarrollo y el abastecimiento de agua de las actividades productivas de los agricultores y los hogares indígenas. Chloe, Artero (2020) Las perturbaciones del sistema de agua 
gestionadas por los usuarios o las comunidades suelen considerarse exógenas, provocadas por la intervención del Estado o del mercado.

La amplia participación de los actores sociales locales en el proceso de toma de decisiones relacionadas con el uso del agua es el factor básico para lograr el desarrollo armónico de la región, porque puede integrar plenamente los conceptos de seguridad hídrica, sustentabilidad y gobernabilidad ambiental democrática, según indica Cortez, (2020).

Según Salazar (2020), proporciona otra perspectiva sobre las percepciones cambiantes del agua en todo el mundo, desde verla como un recurso inagotable hasta verla como finita. Respecto a la región andina, lo sobresaliente es que la gestión es departamental, y está evolucionando hacia una gestión que reconoce que los recursos están integrados, por lo que los usuarios deben actuar en conjunto, lo que también destaca el cambio de espacio de gestión a cuenca en lugar de restricciones políticas. La evolución regulatoria comenzó en la época del Gobernador hasta la Ley de Aguas (1969) incluyó cambios constitucionales que variaron la visión de la gestión de recursos. Asimismo, detalla la brecha entre la realidad y la fecha de publicación (2009) de la L.R.H. en el que manifiesta que el ANA es el ente multisectorial encargado del recurso hídrico, para intervenir, supervisar, crear normas, otorgar derechos de uso de agua, entre otros temas referentes al recurso hídrico, así como los cambios normativos que se han producido en la organización usuaria correspondiente (anteriormente Comité de Usuarios del Distrito de Riego), incluyendo en el medio del Registro de riego. Cambios estrechamente relacionados con el rol que juegan las organizaciones de usuarios (antes organizaciones de riego) en la práctica, como ayudar a resolver conflictos en los recursos nacionales, convirtiéndose en una organización privada, como se ve en este documento.

También es un tema destacado de los usuarios la diferencia entre legislación y realidad. Debido al interés y apoyo obtenido por organizaciones reconocidas a nivel nacional al prestar atención al uso o distribución de los usuarios locales, las organizaciones locales utilizan la gestión ilegal del agua como parte de su organización interna en la comunidad de agricultores. 
Según Martín et al., (2015), indica que la otra cara de lo mismo es el impacto del gran consumo de agua subterránea con fines agrícolas en el uso urbano, al igual que la sobreexplotación del acuífero peruano del Valle de Ica para el cultivo de espárragos, que provocó una fuerte caída en su volumen de agua. Afecta gravemente la oferta de vecinos de la zona. Desacuerdo entre poderes públicos y realidad. Debido a la falta de permisos sociales, judicialización y tensiones regionales, los conflictos relacionados con los recursos hídricos se han convertido en bloqueos de proyectos, que constituyen una realidad real en la mayoría de los países. Si los conflictos hídricos son aislados, accidentales o excepcionales, es imposible continuar con la gestión. Por el contrario, son estructurales, su aumento se debe al fracaso en enfrentar el abandono a largo plazo del problema del agua y el avance del discurso de derechos, que ha generado reclamos y resistencias. El conflicto del agua surge principalmente de una crisis inesperada, junto con el empoderamiento de los grupos vulnerables.

El caso también plantea que cuando las autoridades buscaron hacer cumplir la normativa oficial, además de la informal, no se cumplió la norma oficial final, como propusieron las autoridades, o cuando se reconocieron diferencias, se hizo cumplir. Como explica Villanueva (2016), En su ponencia revisó el proceso de conformación del Comité Interregional de Cuenca Chillón-Rímac-Lurín, en el cual manifestó que el comité de usuarios no accedió a formar el comité, por lo que propuso una organización singular que reconociera la especialidad de sus normas de creación, puede ser sustentada y reconocida de acuerdo a las características de la cuenca que la constituye.

Finalmente, en cuanto a la organización de los usuarios de la gestión del agua, según su ámbito de responsabilidad, la ley prevé tres niveles: (i) junta de usuarios del sector del agua, (ii) comisión de usuarios y (iii) comité de usuarios. En el caso de las cuencas de los ríos Virú y Santa, nos centraremos en la junta de usuarios del sector hidráulico menor de agua de Virú.

Al hablar de gobernanza es embarcarnos en un amplio concepto, como lo demostraron Cruz y Nicandro (2001) en "Gobernanza y gobernabilidad democráticas: conceptos y 
conexiones institucionales confusos y no siempre obvios", el contenido de la "gobernabilidad democrática", en desarrollo, algunas personas señalan que se refiere a la participación de actores políticos, que serán actores gubernamentales y sociales, entendidos como instituciones privadas y ciudadanos. Por tanto, el poder no se ejerce desde el estado, sino para reconocer la participación de personas ajenas al estado.

Casiano, et al. (2016), Destacan la obligación de observar el entorno para definir la gestión del agua, la última visión es muy relevante para prevenir y resolver diferencias o situaciones conflictivas. Si no está seguro del contexto, no puede diseñar una alternativa a la gestión. Con respecto a las cuencas hidrológicas como el campo de gestión de recursos hídricos propuesto por la GIRH, Ortiz e Iván (2008) señalaron en el comité de gestión de cuencas y recursos hídricos que este es un espacio técnicamente suficiente. Además, como parte de la investigación para determinar las herramientas para llegar a un acuerdo, también se considera una definición abierta alternativa de gestión integrada de los recursos hídricos, una de las cuales es integrar los intereses de los usuarios y sus aplicaciones. Y toda la sociedad. Por lo tanto, fortalece el aporte de la gestión de los recursos hídricos en las cuencas hidrológicas a la reducción de conflictos, y deja de lado la gestión departamental, la gestión de las cuencas hidrológicas y la gestión ambiental de los ríos conectados a los centros urbanos. Finalmente, sobre la necesidad de fortalecer los comités de usuarios como participantes en el manejo de las cuencas hidrológicas, demostraron cómo las discusiones sobre el manejo adecuado del agua a nivel latinoamericano llevaron a la necesidad de dar mayor libertad de elección a los diferentes niveles de gobierno. Decisión de autorización de separación de capital o ciudad (sujeto a IWRM). Para este estudio, se puede entender que la gobernanza del agua requiere del consenso de las instituciones y usuarios del agua, en un sentido amplio, teniendo en cuenta los antecedentes de estos participantes. De acuerdo con las necesidades acordadas por los participantes antes mencionados, se revisará si las herramientas determinadas son útiles para la construcción de la gobernanza, o si las diferencias conducen a la división del trabajo en el manejo de cuencas hidrológicas. No cabe duda de que se trata de un hito donde los actores que definen su comportamiento según reglas conviven con otros actores organizados según sus usos y costumbres. 
El Pluralismo legal para Guevara (2008) en "Derechos de agua, pluralismo legal y concreción social" propone ampliar la visión de los derechos del estado cuyo único productor es el estado, a fin de reconocer que la sociedad también produce leyes, que pueden tener mayor legitimidad que las leyes oficiales Normas extraoficiales.

Si bien esta alternativa no es un objetivo directo de la regulación del agua, comprender la LRH y las que se basan en ella desde 2009 permitirá que este poder inherente se ejerza contra organizaciones que aún no han sido reconocidas en la normativa (como las organizaciones de agricultores). La comunidad, porque parte de su función es determinar la distribución del agua. Una muestra de esta situación en el área agrícola de la cuenca del río Viru, donde varias comunidades agrícolas no han sido fusionadas por la Junta de Usuarios del Sector Hidráulico Menor Virú, sin embargo, no han dejado de administrar los recursos hídricos.

Los conflictos según Castellano et. al. (2017) Históricamente, existe evidencia de que, debido a la existencia de múltiples formas de pensar, sentir o expresar ideas, existen diversas disputas entre individuos y grupos sociales.

Según García et. al. (2017) Son muchos los factores internos y externos que inciden en el desarrollo de los conflictos: sujetos conflictivos, sus relaciones, la naturaleza del problema, las condiciones ambientales, la historia del conflicto, etc. A su vez, esto determinará la estrategia a seguir a la hora de resolver el problema: evitación, cooperación o enfrentamiento; y los medios o sistema a utilizar para resolver el conflicto, su naturaleza política o jurídica: negociación, investigación, mediación, reconciliación, arbitraje. , arreglo judicial, integración de nuevos temas. En cualquier caso, se deben seguir dos principios en caso de conflicto: la resolución pacífica del conflicto y la libre elección de los medios para llegar a una solución. La efectividad de este proceso depende de ambos. Naime, (2020), muestra que se están discutiendo dos alternativas: los tomadores de decisiones son racionales, o más precisamente, usan heurística para definir el problema.

Especialmente en lo que respecta a los conflictos por el agua, Huamaní (2015) afirmó que han cambiado, por lo que es necesario capacitar a las instituciones, sus representantes y 
usuarios, y establecer mecanismos para resolver estas situaciones a través del diálogo, en lugar de caer en una situación de crisis. Gobernanza del agua. Destacando que el diálogo es una herramienta consistente de mediación con la GIRH, encaminada a satisfacer los intereses de todas las partes en conflicto; sin embargo, como señalaron Dourojeanni \& Jouralev (1999), no han dejado de reconocer que la gestión de los recursos hídricos es una gestión de conflictos, la herramienta de trabajo se utilizará como referencia para determinar si los actores se apoyan en ella para resolver conflictos o en la práctica que llevó a la creación de herramientas.

La Junta de Usuarios, según indica Ruiz, (2019) Buscan desarrollar estrategias para ayudar a establecer y mantener buenas relaciones con los usuarios a través de nuevas tecnologías de información, orientación y comunicación. El propósito de las recomendaciones anteriores es lograr una gestión institucional eficiente y eficaz, que ayude a diferenciar el uso y la gestión del agua a nivel local y regional. Antecedentes, utilizo la organización en constante cambio para lograr una gran flexibilidad y adaptabilidad al medio ambiente; me esfuerzo por mantener la sostenibilidad y permanecer dentro de su jurisdicción durante mucho tiempo para satisfacer las necesidades de brindar servicios de gestión del agua y buscar el éxito organizacional.

La gestión de Agua, según Villena, (2018) indica que el uso y la gestión del agua deben inspirarse en métodos basados en la participación de usuarios, planificadores y tomadores de decisiones en todos los niveles. Un enfoque participativo significa que los tomadores de decisiones y el público son más conscientes de la importancia del agua. Este enfoque significa que las decisiones deben tomarse en el nivel primario más apropiado, con la consulta pública y la participación de los usuarios en la planificación y ejecución de los proyectos de agua.

Los Usuarios de Agua según el Diccionario de la Real Academia Española (RAE), el usuario es legalmente hablando, de una persona; a través de concesiones gubernamentales u otros derechos legales, puede utilizar aguas de corrientes públicas. La aplicación de la definición de la RAE se refleja en nuestro "Ley de Organizaciones de Usuarios de agua No 30157. Asimismo, en su reglamento, se aprueba por el artículo 3 del Decreto Supremo 005- 
2015-MINAGRI; por lo tanto, para convertirse en usuario de agua en nuestro país, se necesita un derecho de agua otorgado por la Autoridad Nacional del Agua (ANA). No hay diferencia entre usuarios en función del uso del agua. Por lo tanto, todos los usuarios son iguales; con excepción de la escasez, se prioriza el uso poblacional. Sin embargo, en algunos casos, el manejo de recursos compartidos como el agua o los bosques a su alcance requiere que el Estado participe en la supervisión, de esta manera ningún usuario utilizará su condición o condición alguna para lesionar los intereses de otros u otros usuarios. En cuanto a los recursos hídricos, se prevé la organización de estos eventos. En este sentido, las normas mencionadas señalan la naturaleza de las personas naturales sin fines de lucro y las organizaciones de usuarios de agua de personas jurídicas en el artículo 13 y reconocen que existen usuarios con diferentes intereses en el uso del agua porque diferentes sectores productivos usan el agua. Sin embargo, todos deben esforzarse por lograr un uso sostenible de los recursos.

La Autoridad Nacional del Agua según la Ley $\mathrm{N}^{\circ} 29338$, es el ente rector y máxima autoridad técnico- normativa del Sistema Nacional de los Recursos Hídricos, responsable de regular el uso y manejo integral de los recursos hídricos, promoviendo así el uso sostenible, la conservación y el aumento de los recursos hídricos, así como el cumplimiento de las políticas y estrategias nacionales de recursos hídricos y los planes gubernamentales de recursos hídricos en todos los niveles a través de sus órganos desconcertados, Administración Local del Agua (ALA) y Autoridad Administrativa del Agua (AAA).

\section{$\underline{\text { Metodología }}$}

Esta investigación se ha realizado bajo un enfoque cuantitativo, tipo de investigación básica teórica, como señalan Hernández et al., (2003 p. 135), la investigación tiene como objetivo generar nuevo conocimiento mediante la revisión de diferentes teorías. No buscando cambiar la realidad de ninguna manera. Diseño de investigación; Es descriptivo no experimental, transversal o transaccional, porque no produce una situación, sino que observa la situación existente. La investigación no fue provocada deliberadamente por la persona que la llevó a cabo. (Hernández et al., 2003 p. 79). La población o escenario de estudio, se centra en la Junta de Usuarios del Sector Hidráulico Menor Virú que cuenta con 
2151 usuarios, según el padrón interno que maneja esta institución. Se seleccionaron 326 personas para las encuestas por medio del proceso probabilístico de poblaciones finitas.

Como unidad de análisis se tuvo al usuario de la Junta de Usuarios del Sector Hidráulico Menor Virú. Como técnica se consideró el análisis de contenido que puede conducir a la cuantificación de los datos y su posterior interpretación. También se consideró la encuesta como técnica que se utilizó para conocer la percepción de los usuarios respecto a la intervención que realizan los órganos supervisores del estado en la resolución de conflictos por el recurso hídrico, se aplicó mediante un cuestionario físico. Como primer instrumento se utilizó la guía de análisis documental que es un diagnóstico detallado de intervención de los órganos supervisores en la resolución de conflictos de acuerdo con las leyes y normativas vigentes a nivel nacional. El estudio utiliza la gestión y el análisis de la información de forma virtual, con el apoyo del administrador de referencias en línea Mendeley, para archivar información y resaltar aspectos importantes del texto revisado. Otro instrumento se utilizó para evaluar fue el cuestionario físico, con preguntas claras y precisas.

\section{$\underline{\text { Resultados }}$}

Tabla 1.

Variable Intervención en la resolución de conflictos

\begin{tabular}{|c|c|c|c|}
\hline \multicolumn{4}{|c|}{$\begin{array}{l}\text { Intervención de los órganos supervisores del estado en la resolución de conflictos de los } \\
\text { usuarios agrícolas de agua Virú - } 2021\end{array}$} \\
\hline \multirow{5}{*}{ Intervención } & Niveles & f & $\%$ \\
\hline & Deficiente & 0 & $0 \%$ \\
\hline & Regular & 0 & $0 \%$ \\
\hline & Eficiente & 151 & $46 \%$ \\
\hline & Muy eficiente & 175 & $54 \%$ \\
\hline \multicolumn{2}{|c|}{ TOTAL } & 326 & $100 \%$ \\
\hline
\end{tabular}

Nota: Base de datos del instrumento

En tabla $\mathrm{N}^{\circ}$ 1, sobre la variable Intervención de los órganos supervisores en la resolución de conflictos de los usuarios agrarios de agua Virú - 2021, aprecia que 175 
usuarios, que representa el 54\% asegura que la intervención de los órganos supervisores en la resolución de conflictos es muy eficiente; luego 151 usuarios, o sea $46 \%$ señala que tiene un nivel eficiente este campo. La mayoría de los usuarios aseguran que la intervención de los órganos supervisores es muy eficiente y cumple con todos los requerimientos según reglamento.

Tabla 2.

Intervención en la resolución de conflictos por dimensiones

\begin{tabular}{ccccccccc}
\hline \multicolumn{8}{c}{ Intervención de los órganos supervisores en la resolución de conflictos de los usuarios } \\
agrícolas de agua Virú- 2021
\end{tabular}

Nota: Base de datos del instrumento

En la tabla $\mathrm{N}^{\circ} 2$, que se basa en el análisis de la variable intervención en la resolución de conflictos por dimensiones, observamos a la dimensión negociación destinado a la intervención en la resolución de conflictos, señala que el $60 \%$ de los usuarios indican que el nivel de intervención es eficiente, el $22 \%$ en un nivel muy eficiente y el $17 \%$ en un nivel regular; en la dimensión mediación en la intervención el 66\% de los usuarios indican que el nivel es muy eficiente, el 34\% indica que el nivel de intervención es eficiente y solo el $1 \%$ indica que es regular; respecto a la dimensión conciliación el 56\% tiene un nivel muy eficiente, el $43 \%$ un nivel eficiente y el 1\% un nivel regular y en la última dimensión arbitraje en la intervención el $70 \%$ indica que se realiza de manera muy eficiente, el $30 \%$ de nivel eficiente y el $1 \%$ en un nivel regular.

\section{Discusión}

Esta investigación ha formulado una estricta línea metodológica, asumiendo que todas las investigaciones implican un proceso en el uso de métodos científicos para realizar la 
investigación, desde la descripción real de los hechos del trasfondo específico de la intervención hasta la formulación posterior y una serie de etapas hasta los resultados. Se confirman Estos resultados se basan en la meta y se transforman en un análisis la intervención de órganos supervisores del Estado en la resolución de conflictos de los usuarios agrícolas de Agua Virú - 2021. Asimismo, el estudio ha considerado adoptar un diseño de investigación que pueda explicar los resultados obtenidos, de manera que se puedan extraer conclusiones razonables en base a hechos empíricos, de manera que se logre una validez interna y externa dentro del alcance de la investigación. Sujeto a estrictos procedimientos científicos, y los resultados son válidos en un contexto específico, en este caso la Junta del Sector Hidráulico Menor Virú.

En ese sentido es importante indicar que a la luz de los resultados obtenidos y sobre la variable de estudio se ha podido demostrar que la intervención que realizan los órganos supervisores en la resolución de conflictos por el recurso hídrico de los usuarios agrícolas Virú alcanzaron un 54\% en el nivel muy eficiente, y un $46 \%$ en el nivel eficiente, en el que se demuestra que los órganos supervisores como es el ANA (Autoridad Nacional del Agua) y sus anexos los cuales son la Autoridad Administrativa del Agua y la Autoridad Local del Agua cumplen un rol fundamental en la resolución de conflictos por el recurso hídrico dentro de la junta de usuarios de Virú, ya que tienen la facultad de intervenir según la Ley $\mathrm{N}^{\circ} 29338$, donde indica que el ANA es el ente rector y máxima autoridad técnico - normativa del sistema Nacional de Gestión de Recursos Hídricos, añadido a esto también está la Ley $\mathrm{N}^{\circ}$ 30157 que le brinda al ANA la facultad de regular el funcionamiento de las organizaciones de usuarios.

En el objetivo general analizar la Intervención de órganos supervisores del Estado en la resolución de conflictos de los usuarios agrícolas de Agua Virú - 2021, teniendo los datos de la tabla $\mathrm{N}^{\circ} 1$, sobre la variable Intervención de los órganos supervisores en la resolución de conflictos de los usuarios agrarios de agua Virú - 2021, aprecia que 175 usuarios, que representa el 54\% asegura que la intervención de los órganos supervisores en la resolución de conflictos es muy eficiente; luego 151 usuarios, o sea $46 \%$ señala que tiene un nivel 
eficiente este campo. La mayoría de los usuarios aseguran que la intervención de los órganos supervisores es muy eficiente y cumple con todos los requerimientos según reglamento.

Al hacer una comparación con la investigación de Sepulveda et al. (2020) El conflicto de los recursos hídricos debido a la mala calidad y disponibilidad de los recursos ha llevado a un acceso limitado y escaso. Esta situación limita el desarrollo y el abastecimiento de agua de las actividades productivas de los agricultores y los hogares indígenas. Asimismo, Chiloe, Artero (2020) Las perturbaciones del sistema de agua gestionadas por los usuarios o las comunidades suelen considerarse exógenas, provocadas por la intervención del Estado o del mercado, ambos autores indican que hay una mala gestión del recurso hídrico y deficiente participación del ANA.

Según Oré y Rap (2009) en su investigación titulada: Políticas neoliberales de agua en el Perú. Antecedentes y entretelones de la ley de recursos hídricos; la situación actual, indica que el ANA es la máxima autoridad técnico normativa de los recursos hídricos del país, y como ente rector es quien se encarga de intervenir dentro de los conflictos que puedan presentarse por el agua según la norma vigente desde el año 2009 llamada Ley de Recursos hídricos.

Se puede indicar que hasta la fecha el ANA viene realizando una labor muy eficiente dentro del valle de Virú, respecto a la resolución de conflictos de los usuarios agrícolas, puesto que actualmente la Junta de Usuarios se adaptó a la Ley 30157, Ley de organizaciones de usuarios, y la Administración Local del Agua Moche - Virú- Chao, ha sido quien los ha dirigido para la adecuación a la norma vigente.

\section{Conclusiones}

- En esta investigación es importante indicar que a la luz de los resultados obtenidos y sobre la variable de estudio se ha podido demostrar que la intervención que realizan los órganos supervisores en la resolución de conflictos por el recurso hídrico de los usuarios agrícolas Virú alcanzaron un 54\% en el nivel muy eficiente, y un $46 \%$ en el nivel eficiente, en el que se demuestra que los órganos supervisores como es el 
ANA (Autoridad Nacional del Agua) y sus anexos los cuales son la Autoridad Administrativa del Agua y la Autoridad Local del Agua cumplen un rol fundamental en la resolución de conflictos por el recurso hídrico dentro de la junta de usuarios de Virú, ya que tienen la facultad de intervenir según la Ley $\mathrm{N}^{\circ} 29338$, donde indica que el ANA es el ente rector y máxima autoridad técnico - normativa del sistema Nacional de Gestión de Recursos Hídricos, añadido a esto también está la Ley № 30157 que le brinda al ANA la facultad de regular el funcionamiento de las organizaciones de usuarios.

- Se destaca que el ANA como ente encargado del recurso hídrico, realizó negociaciones eficientes teniendo el $60 \%$ de aprobación por parte de los usuarios agrícolas, pero también hay que señalar que aún hay un $17 \%$ que no están conformes con las negociaciones que realizan los órganos desconcertados como son la Administración Local del Agua, Moche- Virú- Chao y la Autoridad Administrativa del Agua, por desconocimiento de la ley de Recursos Hídricos, en los que se debe trabajar para reforzar su capacidad de asesoramiento y negociación.

- Los usuarios agrarios del valle de Virú indicaron que la labor mediadora del ANA es muy eficiente con un $66 \%$ de respaldo, puesto que han visto que el ANA bajo su órgano desconcertado Administración Local del Agua, funciona como ente mediador para la resolución de conflictos por el recurso hídrico, siendo supervisores imparciales, proporcionando estrategias, fomentando el diálogo, mesas de trabajo, y maneja cada conflicto o disputa conforme al marco de la Ley actual vigente.

- Los usuarios agrícolas del valle de Virú destacaron la labor conciliadora que viene realizando el ANA indicando que es muy eficiente con un 56\%, y un $44 \%$ en el nivel eficiente, indicando que usan la capacidad persuasiva, la escucha activa, la manera empática en el trato de los agentes supervisores que a su vez cumplen la labor de conciliador aplicando propuestas de soluciones rápidas y significativas y sobre todo la satisfacción de resolver el conflicto, rigiéndose al marco de la normativa actual.

- Los usuarios agrarios en un 70\% indican que el nivel de arbitraje que realiza el ANA es muy eficiente, eso se debe a que al momento de presentarse ante este organismo, el ALA como órgano desconcertado está predispuesto a intervenir en cualquier 
conflicto o disputa que se presente por el recurso hídrico, ya sea de oficio o mediante una solicitud, usando el método del arbitraje para la solución de controversias, siendo imparcial al momento de aplicar la sentencia o sentencias que se considere necesarios, usando el reglamento de recursos hídricos con conocimiento de causa y las resoluciones que se brindan están bajo la normativa vigente.

\section{$\underline{\text { Referencias }}$}

Altamirano, R; Terreros Lazo, R. (2018) Metodología para determinar la calidad de agua del río Rímac para uso en amasado y curado de concreto. Ingeniería Industrial, núm. 36, Universidad de Lima, Perú. Disponible en: https://www.redalyc.org/articulo.oa?id=337458057007Autoridad Nacional del Agua. (2021) https://www.gob.pe/4404-autoridad-nacional-del-agua-que-hacemos

Autoridad Nacional del Agua. (2021) ¿Qué hacemos? https://www.gob.pe/4404-autoridadnacional-del-agua-que-hacemos

Bonilla, L. (2020). El conflicto social por el acceso al agua: poder y producción de piña en Costa Rica. Revista Espiga, vol. 20, núm. 40. Universidad Estatal a Distancia, Costa Rica. Disponible en: https://www.redalyc.org/articulo.oa?id=467863794005

Barrientos, J. Modelo de gestión integrada de recursos hídricos de las Cuencas de los ríos Moquegua y Tambo. (2011). Universidad de Piura. https://pirhua.udep.edu.pe/bitstream/handle/11042/1480/MAS_GAA_012.pdf

Casiano, C., Gleason, J., Vikolainen, V., Bressers, H. (2016). Evaluación De La Gobernanza: Análisis de la Política de Tratamiento de Aguas Residuales en El Alto Balsas Puebla y Tlaxcala. Disponible en: https://www.researchgate.net/publication/314151813_EVALUACION_DE_LA_G OBERNANZA_ANALISIS_DE_LA_POLITICA_DE_TRATAMIENTO_DE_AG UAS_RESIDUALES_EN_EL_ALTO_BALSAS_PUEBLA_Y_TLAXCALA

Castellano, M., Virviescas Peña, J. Castro Blanco, E., Alvarino Cruz, C., Pinzón Franco, B., Gutiérrez Echeverría, R. (2017). Resolución de conflictos para el fomento de la cultura de paz: importancia de los medios de comunicación alternos en Colombia. Revista Lasallista de Investigación, 14(1),56-65. [fecha de Consulta 1 de octubre de 2021]. ISSN: 1794-4449. Disponible en: https://www.redalyc.org/articulo.oa?id=69551301005 
Cortez, A. (2020). Elementos de conflicto socioambiental: la cervecera Constellation Brands y el agua de Mexicali Frontera Norte, vol. 32, 2020. El Colegio de la Frontera Norte, A.C., México. Disponible en: https://www.redalyc.org/articulo.oa?id=13667947016

Cruz, C. (2001). Gobernabilidad y governance democráticas: el confuso y no siempre evidente vínculo conceptual e institucional. Revista de Agrupación política nueva. Prospectiva. Año 7, número 19. Disponible en: http://www.politicanueva.org.mx

Del Castillo, L. (2008). El régimen legal del agua. En Derechos y conflictos de agua en el Perú, editado por Guevara, A. Departamento Académico de Derecho de la Pontifica Universidad Católica del Perú. (páginas 27 a 50).

http://jornada.pucp.edu.pe/derechodeaguas/wpcontent/uploads/sites/8/2013/07/Texto1.DerechosyConflictosdeAguaenelPeruv2.pdf

Dourojeanni, A. y Jouralev, A. (1999). Gestión de Cuencas y ríos vinculados con centros urbanos. CEPAL- División de Recursos Naturales e Infraestructura. Disponible en: https://repositorio.cepal.org/bitstream/handle/11362/31384/1/S99120968_es.pdf

García, M., Álvarez, T. (2017) La resolución de los conflictos territoriales: reflexiones desde un constitucionalismo global. Gestión y Análisis de Políticas Públicas, núm. 17, mayo, 2017, pp. 63-78. Instituto Nacional de Administración Pública

Guevara, A. (2008). Derechos de Aguas, pluralismo legal y concreción social. En Derechos y conflictos de agua en el Perú, editado por Guevara, A. Departamento Académico de Derecho de la Pontifica Universidad Católica del Perú (páginas 147 a 162). http://jornada.pucp.edu.pe/derecho-de-aguas/wpcontent/uploads/sites/8/2013/07/Texto-1.-

DerechosyConflictosdeAguaenelPeruv2.pdf

Hernández, R., Fernández C. \& Baptista, P. (2003). Metodología de la investigación. México: Mc Graw-Hill Interamericana.

Hernández, R., \& Mendoza Torres, C. P. (2018). Metodología de la investigación: las rutas cuantitativa, cualitativa y mixta. McGraw-Hill Interamericana. https://search.ebscohost.com/login.aspx?direct=true \&db=edselb\&AN=edselb.93398 \&lang=es\&site=eds-live

Huamani, G. (2015). Herramientas para el análisis de los conflictos sociales. En Transformación de conflictos. Aportes al análisis y abordaje de conflictos para el 
desarrollo sostenible, editado por Puma, Liz y Bedoya, César. Asociación Civil ProDiálogo - Prevención y Resolución de Conflictos (páginas 13 a 27). Disponible en:

http://prodialogo.org.pe/sites/default/files/material/files/tranformacion_de_conflicto s.pdf Instituto Nacional de Estadística e Informática. (2014). Compendio estadístico provincia Constitucional del Callao.

https://www.inei.gob.pe/media/MenuRecursivo/publicaciones_digitales/Est/Lib126 8/Glo sario.pdf

Huaricallo, D. Análisis de la gestión del recurso hídrico para consumo humano en la microcuenca Huancho, Huancane, Puno. (2014). Universidad Nacional del Altiplano. Repositorio de tesis - UNA- Puno http://tesis.unap.edu.pe/bitstream/handle/UNAP/2784/Huaricallo_Apaza_David_Co nstantino.pdf?sequence $=1$

Instituto Nacional de Estadística e Informática. (2017). Censo Nacional 2017: XII de Población, VII de Vivienda y III de Comunidades Indígenas. Resultados definitivos. Disponible en Perú: https://www.inei.gob.pe/media/MenuRecursivo/publicaciones_digitales/Est/Lib154 4/

Jara, M. Evaluación de la rentabilidad de los ingresos por uso de agua con fines agrarios en la autoridad local del agua Sicuani periodo 2018. (2019). Universidad Andina del Cusco. https://repositorio.uandina.edu.pe/bitstream/handle/20.500.12557/2932/Mijail_Tesis _bachiller_2019.pdf?sequence=1\&isAllowed=y

López, E., Análisis de la intervención social y calidad del recurso hídrico en el proyecto “Ampliación de los sistemas de agua potable y alcantarillado”, Lurigancho, 2018. (2018). Universidad César Vallejo. https://repositorio.ucv.edu.pe/bitstream/handle/20.500.12692/21323/L\%c3\%b3pez_ GMP.pdf?sequence=1\&isAllowed=y

Martin, L., Bautista, J., (2015). Análisis, prevención y resolución de conflictos por el agua en América Latina y el Caribe. Recursos Naturales e Infraestructura. CEPAL. ISSN 1680-9017. Disponible en https://www.cepal.org/sites/default/files/events/files/analisis_prevencion_y_resoluci on_de_conflictos_por_el_agua_en_america_latina_y_el_caribe_se_ruega_no_circul ar.pdf 
Naime, M. (2020) Defining wicked problems for public policy: The case of Mexico's disappearances. Gestión y Análisis de Políticas Públicas, no. 23, May-October, pp. 64-80. Instituto Nacional de Administración Pública (INAP). Disponible en: https://www.redalyc.org/articulo.oa?id=281563033004.

Oré, Maria T. y Rap, E. (2009). Políticas neoliberales de agua en el Perú. Antecedentes y entretelones de la ley de recursos hídricos. Lima. Debates en Sociología No 34 . Pag. 32 a 66.

Ortiz, I. (2008). Autoridad de cuencas y gestión de recursos hídricos. En Derechos y conflictos de agua en el Perú, editado por Guevara, A. Departamento Académico de Derecho de la Pontifica Universidad Católica del Perú, (páginas 51 a 80). http://jornada.pucp.edu.pe/derecho-de-aguas/wpcontent/uploads/sites/8/2013/07/Texto-1.DerechosyConflictosdeAguaenelPeruv2.pdf

Ruiz, E. (2019). Evaluación ambiental del uso y gestión del agua de riego en la junta de usuarios del sector hidráulico Chira, provincia de Sullana - región Piura. Universidad Nacional de Piura. https://repositorio.unp.edu.pe/bitstream/handle/UNP/1849/DOC-RUI-ROS19.pdf? sequence $=1 \&$ is Allowed $=\mathrm{y}$

Salazar, J. (2020). La interacción de los usuarios en la Junta de Usuarios del Sistema Hidráulico Rímac y la presencia de la Ley de Recursos Hídricos. Pontificia Universidad Católica del Perú. https://tesis.pucp.edu.pe/repositorio/bitstream/handle/20.500.12404/16161/SALAZ AR_SALAS_JEANETTE_MARIBEL_LA_INTERACCION_DE_LOS_USUARIO S_EN_LA_JUNTA_DE_USUARIOS.pdf?sequence=1\&isAllowed=y

Sepulveda, R., Taborda Caro, M., Fuentes Doria, D. (2020). Conflictos por el agua y resiliencia comunitaria en el Bajo Sinú: evidencias de disputas y alternatividad. Utopía y Praxis Latinoamericana, vol. 25, núm. Esp.3. Universidad del Zulia, Venezuela Disponible en:

https://www.redalyc.org/articulo.oa?id=27963600009.DOI:https://doi.org/10.5281/z enodo.3907052

Villanueva, J. (2016). La gobernanza de los recursos hídricos en la Cuenca del Río Lurín en el marco de la creación del Consejo de Recursos Hídricos de Cuenca Chillón, Rímac, Lurín. Pontificia Universidad Católica del Perú. https://tesis.pucp.edu.pe/repositorio/handle/20.500.12404/7159 
Villena J. (2018). Calidad del agua y desarrollo sostenible. Revista Peruana de Medicina Experimental y Salud Publica, 35(2), 304-308.

https://dx.doi.org/10.17843/rpmesp.2018.352.3719

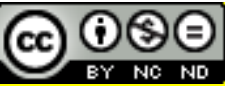

Intervención de órganos del Estado en resolución de conflictos de usuarios de Agua Virú, 2021 (Zurit Fiorela Yakuden - Polonio) Por Revista Sendas se encuentra bajo una Licencia Creative Commons-No Comercial-Sin Derivadas 3.0 Uported. 